\title{
A novel balanced anesthesia shortens time to emergence in patients undergoing modified radical mastectomy: a randomized prospective trial
}

\author{
Linjia Zhu, Zhenfeng Zhang, Sibi Zhang, Xiuhong Jiang, Yan $\mathrm{Ni}^{\wedge}$ \\ Department of Anesthesiology and Perioperative Medicine, The First Affiliated Hospital with Nanjing Medical University, Nanjing, China \\ Contributions: (I) Conception and design: Y Ni; (II) Administrative support: X Jiang; (III) Provision of study materials or patients: L Zhu; (IV) \\ Collection and assembly of data: S Zhang; (V) Data analysis and interpretation: L Zhu, Z Zhang; (VI) Manuscript writing: All authors; (VII) Final \\ approval of manuscript: All authors. \\ Correspondence to: Yan Ni, MD. Department of Anesthesiology and Perioperative Medicine, The First Affiliated Hospital with Nanjing Medical \\ University, 300 Guangzhou Road, Nanjing 210029, China. Email: niyan812@njmu.edu.cn.
}

Background In balanced anesthesia, protocol during the last 30 min is very important to guarantee rapid
emergence and smooth extubation. In clinical practice, sevoflurane and propofol are often used in combination
to achieve a better anesthetic effect and less adverse reaction. Approximately 30 min before surgical completion,
sevoflurane inhalation is often discontinued and propofol is adjusted to keep sufficient depth of anesthesia.
However, propofol-based anesthesia may delay time to emergence due to its unpredictable interindividual
variability. In contrast, sevoflurane can be rapidly excreted unchanged from the respiratory tract, and more
importantly, with minimal variability. This study aimed to investigate the effect of a novel balanced anesthesia
protocol, that is propofol-based intravenous induction, propofol-sevoflurane combined maintenance, and total
sevoflurane inhalation during the last 30 min of the surgery, on the time to emergence/extubation.

Methods: In our study, a total of 100 female patients undergoing modified radical mastectomy were enrolled. All patients received propofol-based intravenous anesthesia for induction followed by propofolsevoflurane combined maintenance. Approximately $30 \mathrm{~min}$ before the end of surgery, sevoflurane was continually inhaled without propofol infusion in group $\operatorname{Sev}(n=50)$, while propofol was only infused in group Pro ( $\mathrm{n}=50$ ). The primary outcome was the time to emergence/extubation. The second outcomes included time to respiratory recovery, and duration of post-anesthesia care unit (PACU) stay. The hemodynamic parameters and incidences of postoperative adverse events such as hypoxemia, nausea, vomiting, dizziness, and emergence agitation (EA) were also assessed.

Results: The time to emergence/extubation in group Sev was shorter than that in group Pro $(12.74 \pm 4.31$ vs. $17.74 \pm 4.27 \mathrm{~min}, \mathrm{P}<0.0001)$. Similarly, time to respiratory recovery, and duration of PACU stay were significantly shortened in group Sev (all $\mathrm{P}<0.0001$ ). Most of the patients in group Sev were extubated under a totally waking state of consciousness. The hemodynamic parameters and incidences of postoperative hypoxemia, nausea, vomiting, dizziness, and EA during the PACU stay were similar between the two groups. Conclusions: In patients undergoing modified radical mastectomy, this novel balanced anesthesia method could shorten the time to emergence/extubation and better waking state without increasing the incidence of adverse events.

Keywords: Balanced anesthesia; emergence/extubation; propofol; sevoflurane; modified radical mastectomy

Submitted Sep 05, 2020. Accepted for publication Nov 15, 2020.

doi: 10.21037/apm-20-1774

View this article at: http://dx.doi.org/10.21037/apm-20-1774

^ORCID: 0000-0001-5228-2677. 


\section{Introduction}

There are approximately 1.5 million women globally diagnosed with breast cancer each year. Modified radical mastectomy, the most common treatment for patients suffering it, is generally performed under general anesthesia (1). Breast cancer exerts a considerable burden on both physical and mental health of the patients (2). Rapid emergence and smooth extubation can decrease the anxiety and restlessness by shortening the interval of separation from family members. In addition, optimization of anesthesia management plays a crucial role in the protocol of enhanced recovery after surgery (3).

In clinical practice, the anesthesia protocol during the last $30 \mathrm{~min}$ is very important to guarantee rapid emergence and smooth extubation. Traditionally, propofol has been used in combination with volatile anesthetics for "balanced anesthesia" regimen (4). Practitioners have been using propofol at induction and during maintenance of anesthesia or in a "sandwich" technique (at induction and again during the last $30 \mathrm{~min}$ before the end of surgery) to reduce postoperative complications, but it may delay emergence and decrease bed turnover rate in post-anesthesia care unit (PACU) (5-8). Previous studies have demonstrated an unpredictable interindividual variability of propofol pharmacokinetics and pharmacodynamics with clinically relevant adverse outcomes (9). The propofol metabolism varies with species, age, physical condition, and the presence or absence of concurrently administered drugs may lead to an unsatisfactory recovery. In contrast, most volatile anesthetics are excreted unchanged by the lungs. Sevoflurane, a widely used major halogenated inhalational anesthetic, has been solely used for anesthesia induction and maintenance in adults and pediatric patients $(10,11)$. In view of its special characteristics of rapid onset and dispersal, sevoflurane can be rapidly expelled from the respiratory tract by increasing the flow of fresh air with minimal variability (12).

We hypothesized that a novel balanced anesthesia method, that is propofol-based induction, propofolsevoflurane combined maintenance, and total sevoflurane inhalation during the last $30 \mathrm{~min}$ of modified radical mastectomy could shorten the time to emergence/ extubation under a totally waking state of consciousness without increasing postoperative complications.

We present the following article in accordance with the CONSORT reporting checklist (available at http://dx.doi. org/10.21037/apm-20-1774).

\section{Methods}

\section{Basic patient data and characteristics}

After obtaining approval from the Institutional Ethics Committee of Jiangsu Province Hospital (the $1^{\text {st }}$ Affiliated Hospital of Nanjing Medical University) (Approval number: 2019-SR-201) and registering in the Chinese clinical trial database (trial registration: ChiCTR1900025320), we recruited 100 female patients who were scheduled for elective modified radical mastectomy in Jiangsu Province Hospital (the $1^{\text {st }}$ Affiliated Hospital of Nanjing Medical University) from June 2019 to March 2020. All patients or their legal representative signed the informed consent form prior to enrollment. Patients aged 18-64 years and American Society of Anesthesiology Physical Status I or II were recruited. Patients with pulmonary insufficiency, muscle relaxant antagonist contraindications, alcohol or drug addiction, obesity (BMI $\geq 30)$, soybean allergy or pregnancy were excluded. After enrollment, the patients were randomized into the sevoflurane group (group Sev, $\mathrm{n}=50$ ) or propofol group (group Pro, $\mathrm{n}=50$ ) (Figure 1). The allocation was performed using a random permuted block method. At admission of PACU, all recovery characteristics were assessed by an anesthesiologist blinded to the trial. The study was conducted in accordance with the Declaration of Helsinki (as revised in 2013).

\section{Anesthesia and emergence}

Anesthesia was administered by the same anesthesiologist. Perioperative patient management was standardized except for anesthesia protocol applied at the last $30 \mathrm{~min}$ of the surgery. All patients fasted before surgery with no premedication. Upon admitting to the operating room, the patients received standard monitoring, such as heart rate (HR), pulse oximetry and electrocardiogram. Invasive arterial pressure was performed on the radial artery opposite to the operating site for continuous blood pressure monitoring. Routine perioperative monitors also included bispectral index monitoring (BIS) and train of four (TOF) stimulation. These indices were measured using a Mindray T6 monitor (Mindray Inc., Shenzhen, China).

All patients in both groups were administered midazolam (Jiangsu Enhua Pharmaceutical Co., Jiangsu, China) $0.05 \mathrm{mg} / \mathrm{kg}$, propofol (AstraZeneca, Corden Pharma S.P.A., Caponago, Italy) $1.5 \mathrm{mg} / \mathrm{kg}$, fentanyl citrate (Yichang Renfu Inc., Yichang, China) $4 \mu \mathrm{g} / \mathrm{kg}$ and cis-atracurium (Shanghai Hengrui Pharmaceutical Ltd., Shanghai, China) $0.2 \mathrm{mg} / \mathrm{kg}$ 
followed by intubation and mechanical ventilation. Ultrasound-guided Pecs II block was performed under complete aseptic technique preoperatively. Throughout the operation, anesthesia was maintained with a continuous intravenous infusion of 2-4 $\mathrm{mg} \cdot \mathrm{kg}^{-1} \cdot \mathrm{h}^{-1}$ propofol (Fresenius Kabi Austria GmbH, Beijing, China) and inhalation of 1-2\% sevoflurane (Shanghai Hengrui Pharmaceutical Ltd., Shanghai, China) to target BIS between 40 and 60 . Before the incision was made, $2 \mu \mathrm{g} / \mathrm{kg}$ fentanyl citrate was administered. Cis-atracurium was continuously infused at a

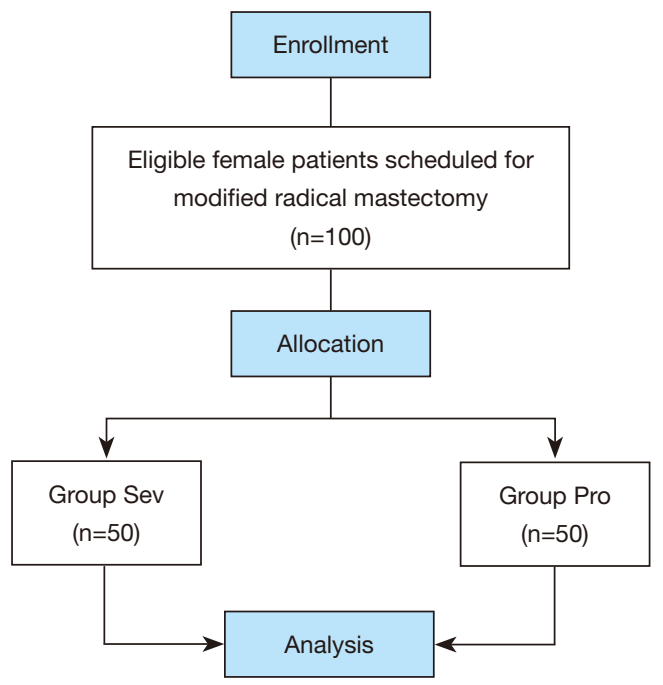

Figure 1 Patient flow chart for the study. A total of 100 (50 in each group) patients scheduled for radical mastectomy were included in our study. rate of $1.5 \mu \mathrm{g} \cdot \mathrm{kg}^{-1} \cdot \mathrm{min}^{-1}$ to maintain TOF count $<2$. Sentinel node biopsy was performed intraoperatively, and patients with sentinel node metastases received completion axillary lymph node dissection. As shown in Figure 2, approximately $30 \mathrm{~min}$ before the end of the surgery, when axillary lymph node was dissected, cis-atracurium was stopped, and total intravenous anesthesia using $4-6 \mathrm{mg} \cdot \mathrm{kg}^{-1} \cdot \mathrm{h}^{-1}$ propofol was performed in group Pro, while patients in group Sev were completely maintained with $1-2 \%$ sevoflurane inhalation. When the surgery was finished, all anesthetics were stopped. All patients were subsequently transferred to the PACU waiting for natural emergence and extubation. Once the TOF count was restored to 2 , neostigmine $(40 \mu \mathrm{g} / \mathrm{kg})$ and atropine $(20 \mu \mathrm{g} / \mathrm{kg})$ were infused to reverse residual muscle blockage effects. When spontaneous breathing resumed, the tidal volume and respiratory rate recovered without causing carbon dioxide accumulation and BIS was $\geq 80$, the nurse anesthetist in the PACU would ask the patients to open their eyes every $5 \mathrm{~min}$. If the patients could cooperate, the tracheal tube would be extubated. When stable vital signs and normal pulse oximetry with regular spontaneous breathing were obtained, the patients were observed with room air breathing until PACU discharge according to our institutional protocol (Aldrete score of $\geq 9$ ) (Figure 2). All patients were generally discharged on the second day after surgery without adverse complaint.

\section{Outcomes}

Patient demographic data, anesthesia time (from anesthesia induction to the end of the surgery), operation time (from

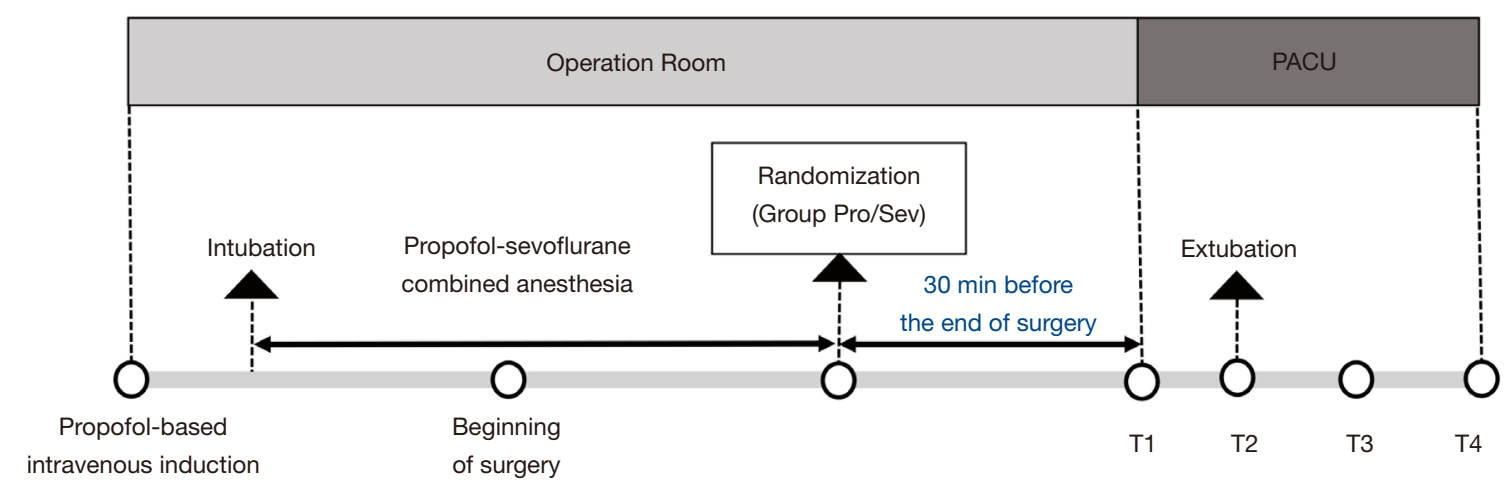

Figure 2 A novel balanced anesthesia protocol. Anesthesia was inducted by propofol-based intravenous anesthetics, and maintained by propofol-sevoflurane combination, followed by total propofol/total sevoflurane anesthesia during the last 30 min of radical mastectomy in group Pro and Sev, respectively. $\mathrm{T}_{1}$ : at admission of PACU; $\mathrm{T}_{2}$ : immediately after extubation; $\mathrm{T}_{3}$ : five minutes after extubation; $\mathrm{T}_{4}$ : ten minutes after extubation. PACU, post-anesthesia care unit. 
Table 1 Patient demographics and baseline characteristics $[\bar{x} \pm \mathrm{SD}$ or $\mathrm{n}(\%)]$

\begin{tabular}{|c|c|c|c|}
\hline Variables & Group Sev $(n=50)$ & Group Pro $(n=50)$ & $\mathrm{P}$ \\
\hline BMI $\left(\mathrm{kg} / \mathrm{m}^{2}\right)$ & $21.97 \pm 3.87$ & $22.81 \pm 3.70$ & 0.27 \\
\hline ASA $(I / I I)$ & $39 / 11$ & $40 / 10$ & 0.81 \\
\hline Hypertension & $28 \%$ & $34 \%$ & 0.22 \\
\hline SLND/CALND & $23 / 27$ & $20 / 30$ & 0.55 \\
\hline Duration of operation (min) & $64.8 \pm 5.2$ & $65.4 \pm 4.9$ & 0.55 \\
\hline Total anesthesia time (min) & $78.8 \pm 6.7$ & $80.4 \pm 7.1$ & 0.25 \\
\hline Anesthesia intervention time (min) & $29.7 \pm 3.7$ & $31.1 \pm 4.9$ & 0.11 \\
\hline
\end{tabular}

Sev, sevoflurane; Pro, propofol; BMI, body mass index; ASA, American Society of Anesthesiologists; SLND, sentinel lymph node dissection; CALND, completion axillary lymph node dissection.

incision to the end of the operation), and anesthesia intervention time (from propofol anesthesia/sevoflurane inhalation anesthesia to the end of the operation) were recorded.

The main measures included the time to emergence/ extubation (from entering PACU to withdrawal of tracheal tube), time to respiratory recovery (from anesthetic cessation to spontaneous breathing recovery) and duration of PACU stay (from admission to discharge of PACU). Hemodynamic parameters including systolic blood pressure (SBP), diastolic blood pressure (DBP), and $\mathrm{HR}$, were documented at admission to the PACU $\left(\mathrm{T}_{1}\right)$ and immediately $\left(\mathrm{T}_{2}\right), 5 \mathrm{~min}\left(\mathrm{~T}_{3}\right)$, and $10 \mathrm{~min}\left(\mathrm{~T}_{4}\right)$ after emergence/extubation. Steward score scale was used to assess levels of consciousness $(0=$ no response to stimuli, $1=$ have response to stimuli, $2=$ fully awake), degree of unobstructed airway $(0=$ respiratory tract needs to be supported, $1=$ unobstructed airway maintained without support, $2=$ coughing according to the physician), and physical activity $(0=$ no physical activity, $1=$ unconsciously performed physical activity, $2=$ consciously performed physical activity) (13). The incidences of intraoperative awareness, hypoxemia $\left(\mathrm{SpO}_{2}<90 \%\right.$ for more than $1 \mathrm{~min}$ under air condition), nausea, vomiting, dizziness, and emergence agitation (EA) after extubation were evaluated. The rescue of antiemetic drugs was also recorded to reflect the occurrence of nausea and vomiting in the ward.

\section{Statistical analysis}

According to the pilot experiment with a sample size of 10 patients in each group, we obtained the emergence/ extubation time in two groups (the mean difference between two groups: $2.2 \mathrm{~min}$ ). For two-tailed statistical analysis, 41 patients were needed in each group with a risk of type-I error of 0.05 and power of 0.8 . To compensate for $20 \%$ possible dropouts, 50 patients were enrolled per group.

All data were collected by a blinded investigator. Statistical analysis and data process were performed using SPSS version 22.0 software (SPSS Inc., IBM Corporation, Armonk, NY, USA) and GraphPad Prism version 8 (GraphPad Software, Inc., San Diego, CA, USA). The Shapiro-Wilk test was used to assess whether the data were normally distributed. Quantitative data that conformed to a normal distribution were expressed as the mean \pm standard deviation (SD) and were compared by independent-samples $t$-tests. Qualitative data were expressed as percentages (\%). Data for patients who experienced complications during their PACU stay were collected and compared using the Pearson chi-square test, continuous calibration chi-square test or Fisher's test, as appropriate. $\mathrm{P}<0.05$ was considered statistically significant.

\section{Results}

A total of 100 female patients scheduled for modified radical mastectomy between June 2019 and March 2020 in our hospital were included in our study. The patients' demographic data were comparable between the two groups ( $\mathrm{P}>0.05$, Table 1).

As shown in Figure 3, the time to emergence/ extubation in group $\mathrm{Sev}$ was significantly shorter than that in group Pro $(12.74 \pm 4.31$ vs. $17.74 \pm 4.27 \mathrm{~min}$, 
$\mathrm{P}<0.0001)$. The time to spontaneous breath recovery and duration of PACU stay in group Sev were obviously decreased than those in group Pro $(10.54 \pm 4.65$ vs. $15.54 \pm 4.19 \mathrm{~min}$, $41.04 \pm 5.54$ vs. $49.24 \pm 5.58 \mathrm{~min}$, all $\mathrm{P}<0.0001$, Figure 4). No obvious differences were observed in SBP, DBP, and HR at different time points during the PACU stay between the two groups (Figure 5). At the time points of $\mathrm{T}_{2}-\mathrm{T}_{4}$, the levels of consciousness were all higher in group Sev than

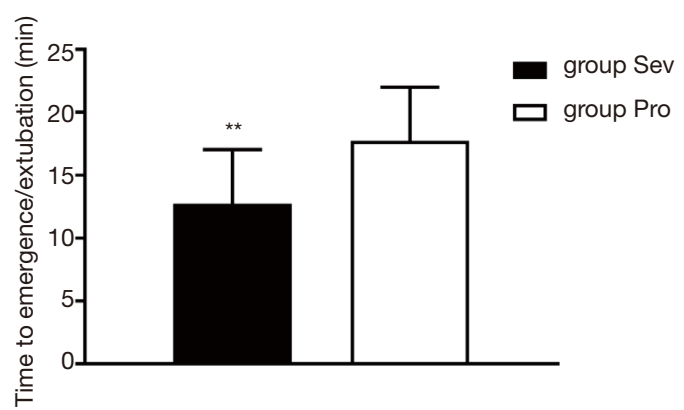

Figure 3 Time to emergence/extubation in the both groups. Variables are expressed as means $\pm \mathrm{SD},{ }^{* *} \mathrm{P}<0.0001$ vs. group Pro.

A

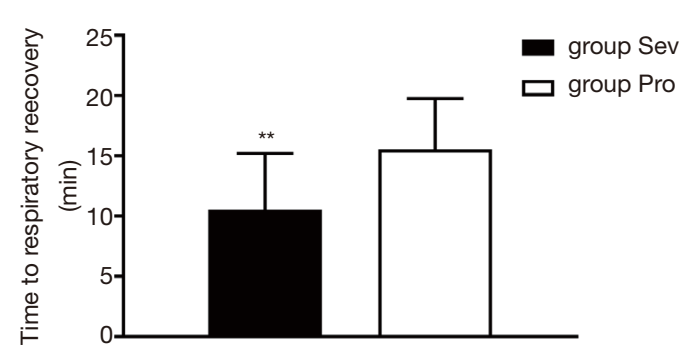

those of group Pro (all $\mathrm{P}=0.001$ ). Similarly, the degrees of unobstructed airway were better in group $\mathrm{Sev}$ at $\mathrm{T}_{2}$ and $\mathrm{T}_{3}$ (all $\mathrm{P}=0.002$ ), but no difference at $\mathrm{T}_{4}$. The physical activity cooperation was better in group Sev at $\mathrm{T}_{2}(\mathrm{P}=0.004)$, but five and ten minutes after extubation, it was comparable between the two groups (Table 2). No significant differences were found in the occurrence of dizziness, hypoxemia, nausea and vomiting, and EA between the two groups (Table 3).

\section{Discussion}

As a malignant tumor with high morbidity and mortality, breast cancer has become the leading cause of cancerrelated death among women $(14,15)$; similarly, in China, it was estimated that 278,000 new cases occurred in 2013, and the mortality rate reached $6.95 \%(16,17)$. Clinically, modified radical mastectomy remains the mainstream therapy, and stable anesthesia, effective analgesia, and highquality recovery are critical factors that affect postoperative rehabilitation. Different anesthesia protocols and anesthetics may have distinct influences on emergence/extubation time

B

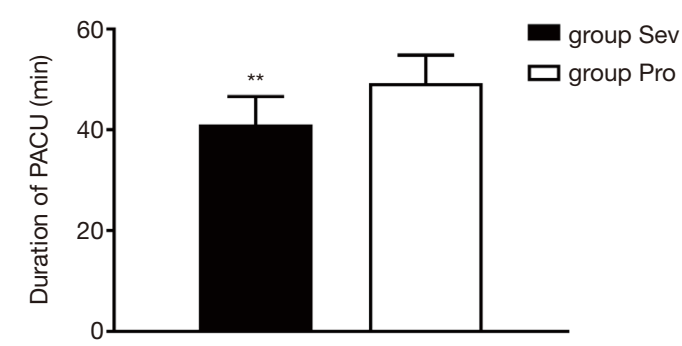

Figure 4 Time to spontaneous breath recovery, time to emergence, and duration of PACU stay of both groups. Variables are expressed as means $\pm \mathrm{SD},{ }^{* *} \mathrm{P}<0.0001$ vs. group Pro.
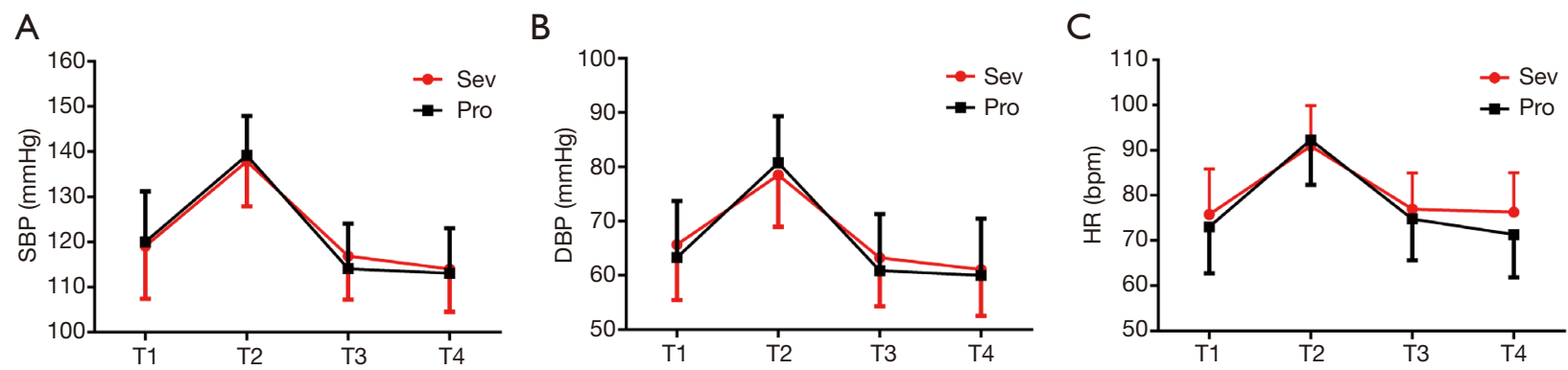

Figure 5 Hemodynamic parameter changes at four different time points in the two groups. (A) SBP; (B) DBP; (C) HR. Variables are expressed as means $\pm S D$. $T_{1}$ : baseline; $T_{2}$ : immediately after extubation; $T_{3}$ : five minutes after extubation; $T_{4}$ : ten minutes after extubation. SBP, systolic blood pressure; DBP, diastolic blood pressure; HR, heart rate. 
Table 2 The response of patients after extubation using Steward score [n (\%)]

\begin{tabular}{|c|c|c|c|}
\hline Steward score & Group Sev $(n=50)$ & Group Pro $(n=50)$ & $\mathrm{P}$ \\
\hline Consciousness (0/1/2) & $0 / 5 / 45$ & $0 / 20 / 30$ & $0.001^{* *}$ \\
\hline Degree of airway $(0 / 1 / 2)$ & $0 / 3 / 47$ & $0 / 15 / 35$ & $0.002^{\star \star}$ \\
\hline Physical activity $(0 / 1 / 2)$ & $0 / 1 / 49$ & $0 / 10 / 40$ & $0.004^{* *}$ \\
\hline Consciousness $(0 / 1 / 2)$ & $0 / 3 / 47$ & $0 / 16 / 34$ & $0.001^{\star \star}$ \\
\hline Degree of airway $(0 / 1 / 2)$ & $0 / 1 / 49$ & 0/11/39 & $0.002^{\star \star}$ \\
\hline Physical activity (0/1/2) & $0 / 0 / 50$ & $0 / 5 / 45$ & 0.066 \\
\hline \multicolumn{4}{|l|}{$\mathrm{T}_{4}$} \\
\hline Physical activity (0/1/2) & $0 / 0 / 50$ & $0 / 1 / 49$ & 1.000 \\
\hline
\end{tabular}

${ }^{* *} \mathrm{P}<0.01$, compared with group Pro. Sev, sevoflurane; Pro, propofol; consciousness $(0=$ no response to stimuli, $1=$ have response to stimuli, $2=$ fully awake), degree of airway $(0=$ need manual support to keep airway open, $1=$ no need for support, $2=$ cough according to verbal command), and physical activity $(0=$ no physical activity, $1=$ unconsciously performed physical activity, $2=$ consciously performed physical activity).

Table 3 Complications during the procedure and recovery period [n (\%)]

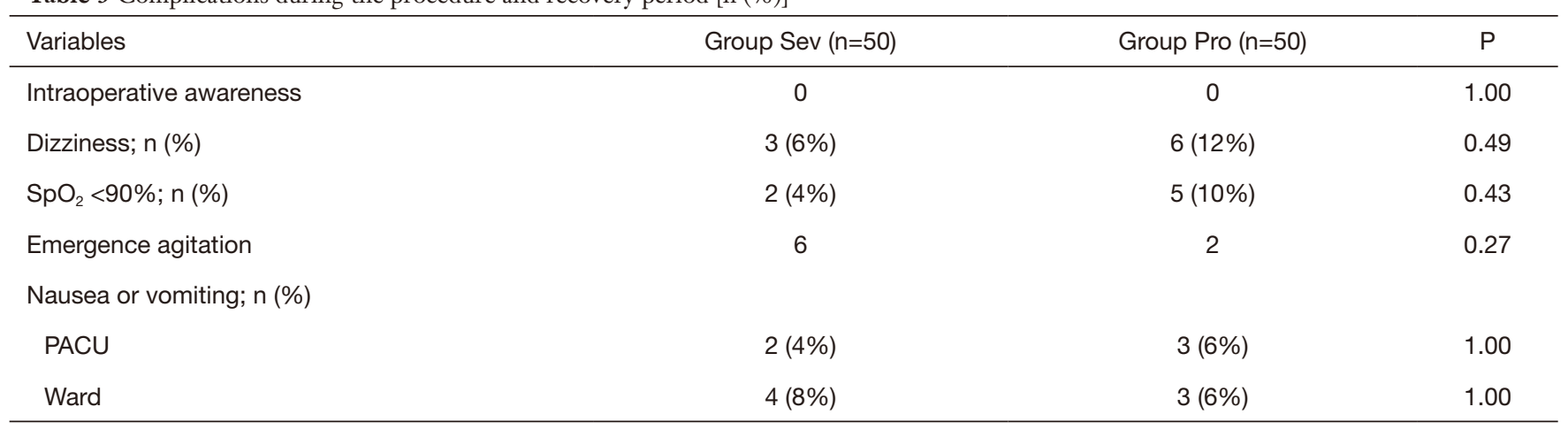

Sev, sevoflurane; Pro, propofol; PACU, post-anesthesia care unit.

and subsequently affect postoperative recovery quality. As our study demonstrated, this novel balanced-anesthesia method could effectively provide a faster emergence/ extubation and better waking state without increasing postoperative complications.

Propofol is currently the most frequently used intravenous anesthetic on account of its rapid recovery profile. However, after prolonged high-dose propofol infusion, the plasma level of propofol may decrease slowly and its half time may increase more $(5,18)$. Furthermore, compared with sevoflurane, propofol is metabolized dependent of liver and renal function; therefore, it is less controllable. Tang et al. reported that patients receiving propofol-based intravenous anesthesia experienced a delay in the decided extubation time compared with the sevoflurane group (19), which is in accordance with our results. In contrast, of all currently used anesthetics, the pharmacokinetic properties of sevoflurane come closest to that of the ideal anesthetic. Sevoflurane, an inhalational anesthetic, has the advantages of low solubility in blood and tissues and can be dissipated quickly and completely at the end of surgery by increasing the flow of fresh gas, 
even at a high concentration (20). In previous study, Bocskai et al. found that the time to extubation after total sevoflurane anesthesia was approximately $11-15 \mathrm{~min}$, which is similar with our findings (20). Hepağuşlar et al. showed that patients under sevoflurane anesthesia were more alert and ready to go home earlier than those under propofolbased general anesthesia (21), which is in accordance with our results. Gupta et al. also demonstrated that sevoflurane added a newer dimension to recovery by allowing rapid emergence and early discharge (22). All the results above are in agreement with ours.

Over the course of the PACU stay, no patients suffered with unstable hemodynamic fluctuation. Many investigators have concluded that propofol could effectively prevent cardiovascular and stress responses during the emergence and extubation period (23), which is in accordance with our results. We also found that sevoflurane dissipation would not deteriorate hemodynamics during PACU. Some investigators have suggested that propofol could reduce the incidence of perioperative nausea and vomiting $(24,25)$. They pointed out that most postoperative adverse events in the PACU, including nausea, vomiting, somnolence and confusion, were associated with sevoflurane inhalation (26-28). In our present study, we found that our novel balanced anesthesia did not increase the incidence of postoperative nausea and vomiting. The inconsistence between ours and those previous studies might be related to the prophylactic administration of anti-emetic drugs. Although many previous studies have shown that the incidence of EA is closely related to sevoflurane inhalation $(29,30)$, only a few patients in both groups suffered from EA. This result is in accordance with the findings of other investigators who showed that the incidence of post-anesthesia agitation, postoperative outcomes, and complications were not significantly different between the TCI propofol and sevoflurane groups during kidney transplant surgery (31). In our study, the concentration of sevoflurane was adjusted between $1 \%$ and $2 \%$, which may be associated with the low rate of EA. The result is exactly as Feng et al. implied, that the incidence of EA during recovery was independent of different inhaled sevoflurane concentrations (within 1.5 MAC) in adults (32). In addition, propofol was confirmed to prevent agitation after sevoflurane anesthesia in children (33). In our study, during the anesthesia maintenance, we applied propofol-sevoflurane combined anesthesia rather than total sevoflurane inhalation, which may have had a preventive effect against the nausea, vomiting and EA associated with propofol.
Several limitations should be taken into account when interpreting the results. First, we performed ultrasoundguided Pecs II block preoperatively, and only one patient complained of moderate pain during the PACU stay, we did not assess visual analogue score. Second, we simply observed the incidence of nausea and vomiting in PACU and in ward without comparing severity grades. Third, although breast surgery is traditionally performed under general anesthesia, the use of locoregional anesthesia techniques, including thoracic paravertebral block, pectoral nerve block, erector spinae plane block, and serratus anterior plane block, have been successfully implemented in many breast surgeries $(34,35)$. We did not compare the advantages and disadvantages between regional anesthesia and general anesthesia. Therefore, a further prospective randomized study is needed to determine the factors that impact outcomes in patients receiving similar anesthesia schemes.

In conclusion, the application of a novel anesthesia method with propofol-based intravenous induction and propofol-sevoflurane combined maintenance, followed by total sevoflurane inhalation during the last $30 \mathrm{~min}$ of radical mastectomy, could provide an accelerated emergence/extubation and better waking state from general anesthesia without increasing the incidence of postoperative complications.

\section{Acknowledgments}

Funding: None.

\section{Footnote}

Reporting Checklist: The authors have completed the CONSORT reporting checklist. Available at http://dx.doi. org/10.21037/apm-20-1774

Data Sharing Statement: Available at http://dx.doi. org/10.21037/apm-20-1774

Peer Review File: Available at http://dx.doi.org/10.21037/apm20-1774

Conflicts of Interest: All authors have completed the ICMJE uniform disclosure form (available at http://dx.doi. org/10.21037/apm-20-1774). The authors have no conflicts of interest to declare.

Ethical Statement: The authors are accountable for all 
aspects of the work in ensuring that questions related to the accuracy or integrity of any part of the work are appropriately investigated and resolved. The study was conducted in accordance with the Declaration of Helsinki (as revised in 2013). This study was approved by the Institutional Ethics Committee of Jiangsu Province Hospital (the $1^{\text {st }}$ Affiliated Hospital of Nanjing Medical University) (No.: 2019-SR-201) and was registered in the Chinese clinical trial (No.: ChiCTR1900025320). All patients or their legal representative signed the informed consent forms.

Open Access Statement: This is an Open Access article distributed in accordance with the Creative Commons Attribution-NonCommercial-NoDerivs 4.0 International License (CC BY-NC-ND 4.0), which permits the noncommercial replication and distribution of the article with the strict proviso that no changes or edits are made and the original work is properly cited (including links to both the formal publication through the relevant DOI and the license). See: https://creativecommons.org/licenses/by-nc-nd/4.0/.

\section{References}

1. Tokgöz S, Umay EK, Yilmaz KB, et al. Role of intraoperative nerve monitoring in postoperative muscle and nerve function of patients undergoing modified radical mastectomy. J Invest Surg 2019:1-8.

2. Wu G, Fu G, Zhang L, et al. Effects of neoadjuvant chemotherapy on the depth of total intravenous anesthesia in patients with breast cancer undergoing unilateral modified radical mastectomy: A prospective observational study. Medicine (Baltimore) 2018;97:e13776.

3. Melnyk M, Casey RG, Black P, et al. Enhanced recovery after surgery (ERAS) protocols: Time to change practice? Can Urol Assoc J 2011;5:342-8.

4. Dinis-Oliveira RJ. Metabolic profiles of propofol and fospropofol: Clinical and forensic interpretative aspects. Biomed Res Int 2018;2018:6852857.

5. Omara AF, Abdelrahman AF, Elshiekh ML. Recovery with propofol anesthesia in children undergoing cleft palate repair compared with sevoflurane anesthesia. Anesth Pain Med 2019;9:e92076.

6. Weissman C, Scemama J, Weiss YG. The ratio of PACU length-of-stay to surgical duration: Practical observations. Acta Anaesthesiol Scand 2019;63:1143-51.

7. Jellish WS, Leonetti JP, Fahey K, et al. Comparison of 3 different anesthetic techniques on 24-hour recovery after otologic surgical procedures. Otolaryngol Head Neck Surg 1999;120:406-11.

8. Soppitt AJ, Glass PS, Howell S, et al. The use of propofol for its antiemetic effect: A survey of clinical practice in the United States. J Clin Anesth 2000;12:265-9.

9. Loryan I, Lindqvist M, Johansson I, et al. Influence of sex on propofol metabolism, a pilot study: Implications for propofol anesthesia. Eur J Clin Pharmacol 2012;68:397-406.

10. Chung PK, Dhanrajani P. Comparative randomized study of propofol target-controlled infusion versus sevoflurane anesthesia for third molar extraction. J Dent Anesth Pain Med 2018;18:169-75.

11. Mo L, Hong S, Li Y, et al. Sevoflurane inhibited inflammatory response induced by TNF- $\alpha$ in human trophoblastic cells through p38MAPK signaling pathway. J Recept Signal Transduct Res 2020;40:218-23.

12. White PF. Facilitating recovery from anesthesia: Assessing the costs and benefits of anesthetic drugs. Anesth Analg 2010;110:273-5.

13. Zhao X, Jin YW, Li HB, et al. Effects of maintaining intravenous infusion of remifentanil or propofol on anesthesia and palinesthesia during anesthesia and analepsia. Genet Mol Res 2014;13:2865-72.

14. Tavare AN, Perry NJS, Benzonana LL, et al. Cancer recurrence after surgery: Direct and indirect effects of anesthetic agents. Int J Cancer 2012;130:1237-50.

15. Siegel RL, Miller KD, Jemal A. Cancer statistics, 2018. CA Cancer J Clin 2018;68:7-30.

16. Chen $\mathrm{W}$, Zheng R, Baade PD, et al. Cancer statistics in China, 2015. CA Cancer J Clin 2016;66:115-32.

17. Zheng R, Zeng H, Zhang S, et al. Estimates of cancer incidence and mortality in China, 2013. Chin J Cancer 2017;36:66.

18. Hachenberg T. Perioperative management with shortacting intravenous anesthetics. Anaesthesiol Reanim 2000;25:144-50.

19. Tang L, Liu H, Wu Y, et al. Sevoflurane may be more beneficial than propofol in patients receiving endoscopic variceal ligation and endoscopic variceal sclerotherapy: A randomized, double-blind study. Exp Ther Med 2017;14:3145-52.

20. Bocskai T, Loibl C, Vamos Z, et al. Cost-effectiveness of anesthesia maintained with sevoflurane or propofol with and without additional monitoring: A prospective, randomized controlled trial. BMC Anesthesiol 2018;18:100.

21. Hepağuşlar H, Ozzeybek D, Ozkardeşler S, et al. Propofol and sevoflurane during epidural/general anesthesia: 
Comparison of early recovery characteristics and pain relief. Middle East J Anaesthesiol 2004;17:819-32.

22. Gupta A, Stierer T, Zuckerman R, et al. Comparison of recovery profile after ambulatory anesthesia with propofol, isoflurane, sevoflurane and desflurane: A systematic review. Anesth Analg 2004;98:632.

23. Janardhana VK, Thimmaiah V. A prospective, randomized, single-blind, comparative study of dexmedetomidine and propofol infusion for intraoperative hemodynamics and recovery characteristics in laparoscopic surgeries. Anesth Essays Res 2019;13:492-7.

24. Matsuura H, Inoue S, Kawaguchi M. The risk of postoperative nausea and vomiting between surgical patients received propofol and sevoflurane anesthesia: A matched study. Acta Anaesthesiol Taiwan 2016;54:114-20.

25. Çaparlar CÖ, Özhan MÖ, Süzer MA, et al. Fast-track anesthesia in patients undergoing outpatient laparoscopic cholecystectomy: Comparison of sevoflurane with total intravenous anesthesia. J Clin Anesth 2017;37:25-30.

26. Lee DW, Lee HG, Jeong CY, et al. Postoperative nausea and vomiting after mastoidectomy with tympanoplasty: A comparison between TIVA with propofol-remifentanil and balanced anesthesia with sevoflurane-remifentanil. Korean J Anesthesiol 2011;61:399-404.

27. Saros GB, Doolke A, Anderson RE, et al. Desflurane vs. sevoflurane as the main inhaled anaesthetic for spontaneous breathing via a laryngeal mask for varicose vein day surgery: A prospective randomized study. Acta Anaesthesiol Scand 2006;50:549-52.

28. Chung F, Mulier JP, Scholz J, et al. A comparison of anaesthesia using remifentanil combined with either isoflurane, enflurane or propofol in patients undergoing

Cite this article as: Zhu L, Zhang Z, Zhang S, Jiang X, Ni Y. A novel balanced anesthesia shortens time to emergence in patients undergoing modified radical mastectomy: a randomized prospective trial. Ann Palliat Med 2021;10(3):2439-2447. doi: 10.21037/apm-20-1774 gynaecological laparoscopy, varicose vein or arthroscopic surgery. Acta Anaesthesiol Scand 2000;44:790-8.

29. Kocaturk O, Keles S. Recovery characteristics of total intravenous anesthesia with propofol versus sevoflurane anesthesia: A prospective randomized clinical trial. J Pain Res 2018;11:1289-95.

30. Omara AF, Abdelrahman AF, Elshiekh ML. Recovery with propofol anesthesia in children undergoing cleft palate repair compared with sevoflurane anesthesia. Anesth Pain Med 2019;9:e92076.

31. Aditianingsih D, Sukmono B, Agung TA, et al. Comparison of the effects of target-controlled infusion of propofol and sevoflurane as maintenance of anesthesia on hemodynamic profile in kidney transplantation. Anesthesiol Res Pract 2019;2019:5629371.

32. Feng Y, Chen XB, Yuan WG, et al. Comparison of the level of free hexafluoro-isopropanol in adults' blood and the incidence of emergence agitation after anesthesia with different concentrations of sevoflurane in laparoscopic gastrointestinal surgery: A randomized controlled clinical trial. Clin Ther 2019;41:2263-72.

33. Wu X, Cao J, Shan C, et al. Efficacy and safety of propofol in preventing emergence agitation after sevoflurane anesthesia for children. Exp Ther Med 2019;17:3136-40.

34. Garreffa E, Cantagalli F, Mastrantuono A, et al. Pectoral nerves blocks (PECS) and sedation: A way to avoid general anesthesia in breast surgery - A single institution early experience. Breast J 2020;26:303-5.

35. Santonastaso DP, de Chiara A, Russo E, et al. A possible future for anaesthesia in breast surgery: Thoracic paravertebral block and awake surgery. A prospective observational study. Tumori 2020:300891620951626. 